\title{
Fusion of optical coherence tomographic and angiographic data for more accurate evaluation of the endothelial shear stress patterns and neointimal distribution after bioresorbable scaffold implantation: comparison with intravascular ultrasound-derived reconstructions
}

\author{
Christos V. Bourantas - Michail I. Papafaklis - Lampros Lakkas • \\ Antonis Sakellarios • Yoshinobu Onuma • Yao-Jun Zhang • Takashi Muramatsu • \\ Roberto Diletti • Paschalis Bizopoulos • Fanis Kalatzis • Katerina K. Naka • \\ Dimitrios I. Fotiadis • Jin Wang • Hector M. Garcia Garcia • Takeshi Kimura • \\ Lampros K. Michalis • Patrick W. Serruys
}

Received: 10 October 2013/Accepted: 15 January 2014/Published online: 24 January 2014

(C) Springer Science+Business Media Dordrecht 2014

\begin{abstract}
Intravascular ultrasound (IVUS)-based reconstructions have been traditionally used to examine the effect of endothelial shear stress (ESS) on neointimal formation. The aim of this analysis is to compare the association between ESS and neointimal thickness (NT) in models obtained by the fusion of optical coherence tomography (OCT) and coronary angiography and in the
\end{abstract}

Christos V. Bourantas and Michail I. Papafaklis have contributed equally to this work.

C. V. Bourantas · Y. Onuma - Y.-J. Zhang · T. Muramatsu R. Diletti · H. M. Garcia Garcia · P. W. Serruys $(\bowtie)$ Department of Interventional Cardiology, Thoraxcenter, Erasmus University Medical Centre, 's-Gravendijkwal 230, 3015 CE Rotterdam, The Netherlands

e-mail: p.w.j.c.serruys@erasmusmc.nl

M. I. Papafaklis

Cardiovascular Division, Brigham and Women's Hospital,

Harvard Medical School, Boston, MA, USA

L. Lakkas · K. K. Naka · L. K. Michalis

Department of Cardiology, Medical School, University of

Ioannina, Ioannina, Greece

A. Sakellarios · P. Bizopoulos · F. Kalatzis - D. I. Fotiadis Department of Materials Science and Engineering, University of Ioannina, Ioannina, Greece

J. Wang

Abbott Vascular, Diegem, Belgium

T. Kimura

Department of Cardiovascular Medicine, Kyoto University

Graduate School of Medicine, Kyoto, Japan reconstructions derived by the integration of IVUS and coronary angiography. We analyzed data from six patients implanted with an Absorb bioresorbable vascular scaffold that had biplane angiography, IVUS and OCT investigation at baseline and 6 or 12 months follow-up. The IVUS and OCT follow-up data were fused separately with the angiographic data to reconstruct the luminal morphology at baseline and follow-up. Blood flow simulation was performed on the baseline reconstructions and the ESS was related to NT. In the OCT-based reconstructions the ESS were lower compared to the IVUS-based models $(1.29 \pm 0.66$ vs. $1.87 \pm 0.66 \mathrm{~Pa}, P=0.030)$. An inverse correlation was noted between the logarithmic transformed ESS and the measured NT in all the OCT-based models which was higher than the correlation reported in five of the six IVUS-derived models $(-0.52 \pm 0.19 \mathrm{~Pa}$ vs. $-0.10 \pm 0.04, P=0.028$ ). Fusion of OCT and coronary angiography appears superior to IVUS-based reconstructions; therefore it should be the method of choice for the study of the effect of the ESS on neointimal proliferation.

Keywords Endothelial shear stress - Neointimal formation - Optical coherence tomography - Bioresorbable scaffold

\section{Introduction}

Until recently, the in vivo evaluation of ESS distribution and its effect on neointimal formation relied on the fusion of coronary angiographic and intravascular ultrasound 
(IVUS) data. However, IVUS is an imaging modality with limited resolution which does not permit meticulous evaluation of luminal morphology and assessment of the hemodynamic micro-environment [1-4]. Frequency domain optical coherence tomography (FD-OCT) is a relatively new technique that provides high resolution crosssectional images which enable detailed study of stented segments as they allow precise evaluation of edge dissection, struts coverage and apposition [5]. FD-OCT has great value in assessing coronary arteries treated with poly-Llactic based bioresorbable scaffolds as the struts of these devices appear as echolucent boxes allowing evaluation of their location with regards to the vessel wall.

We have recently proposed a methodology that enables fusion of FD-OCT and coronary angiographic data and validated this approach in native coronaries using IVUSderived reconstructions as the accepted standard [6, 7]. However, it remains unclear what the incremental value is for FD-OCT-based over the IVUS-based coronary reconstruction in assessing the local ESS patterns and their impact on vessel wall healing in treated coronary arteries. The aim of this study is to compare the ESS, the neointimal distribution and the association between ESS and neointimal thickness (NT) in FD-OCT-based versus IVUS-based reconstructions of scaffolded segments. We aim to highlight the indigenous limitations of the conventional approach in modeling treated segments and in studying the effect of the local hemodynamic milieu on neointimal formation.

\section{Methods}

Included patients and study design

We analyzed data from the patients recruited in the ABSORB Cohort B Trial (A Clinical Evaluation of the Everolimus Eluting Bioresorbable Vascular Scaffold System in the Treatment of Patients with de Novo Native Coronary Artery Lesions) (NCT00856856). The design of this prospective multicenter single arm study has been described in detail by Serruys et al. [8]. In brief, 101 patients with single or 2 vessel de novo coronary disease implanted with an Absorb BVS (Abbott Vascular, Santa Clara, CA, USA) (dimensions: $3.0 \times 18 \mathrm{~mm}$ ) were included in this trial. The studied population was divided in 2 groups and both were investigated with serial angiographic, grayscale IVUS, IVUS virtual histology, and OCT (OCT was an optional examination). The first group (B1) had these tests at baseline post device implantation, 6 months and 2 years follow-up, and the second (B2) at baseline, 1 year and 3 years follow-up. The current analysis included only the patients who had biplane coronary angiography at 6 months or 12 months follow-up and serial IVUS and FD-OCT examinations at baseline and follow-up.
The ABSORB Cohort B study was sponsored and financially supported by Abbott Vascular. The trial was approved by the human research committee of the institutions that participated. Written informed consent was obtained from all patients.

Data acquisition

Coronary angiography was performed with a Siemens Axiom-Artis biplane (Siemens, Erlangen, Germany) system. Two end-diastolic biplane angiographic images acquired during contrast agent injection at 6 (for the patients included in the ABSORB Cohort B1 group) or 12 months (for the patients included in the ABSORB Cohort B2 group) follow-up were selected and used for coronary reconstruction.

IVUS examination was undertaken in each scaffolded segment at baseline immediately after scaffold implantation and at follow-up by a phased-array IVUS catheter (EagleEye, Volcano Corp, Rancho Cordova, CA, USA) with the use of an automated pull-back device which withdrew the catheter at a speed of $0.5 \mathrm{~mm} / \mathrm{s}$. FD-OCT image acquisition was performed at baseline (immediately after scaffold implantation) and at follow-up using a C7XR Fourier Domain system (Lightlab Imaging, Westford, Massachusetts, USA) that was pulled-back at a speed of $20 \mathrm{~mm} / \mathrm{s}$. The acquired data were stored in DICOM format and analyzed offline by three expert observers (CVB, MIP, LL) blinded to patients' procedural and clinical characteristics.

\section{Data processing}

The angiographic, IVUS and FD-OCT data acquired at follow-up were reviewed by the experts who identified the scaffolded segment and two anatomical landmarks (i.e., side branches), one located proximally and the other distally to the scaffolded segment, that were visible in coronary angiography and both intravascular imaging examinations.

The segment of interest, defined by the anatomical landmarks, was further processed in IVUS and FD-OCT images. In particular, the observers reviewed the baseline IVUS sequence and then analyzed the follow-up data where they identified the end-diastolic images and detected the luminal and the scaffold (in the scaffolded segment) borders. Since all the struts at baseline IVUS examination appeared embedded into the vessel wall the scaffold borders at follow-up (which represent the luminal borders at baseline) were defined by splines connecting the adluminal side of the struts (Fig. 1). 

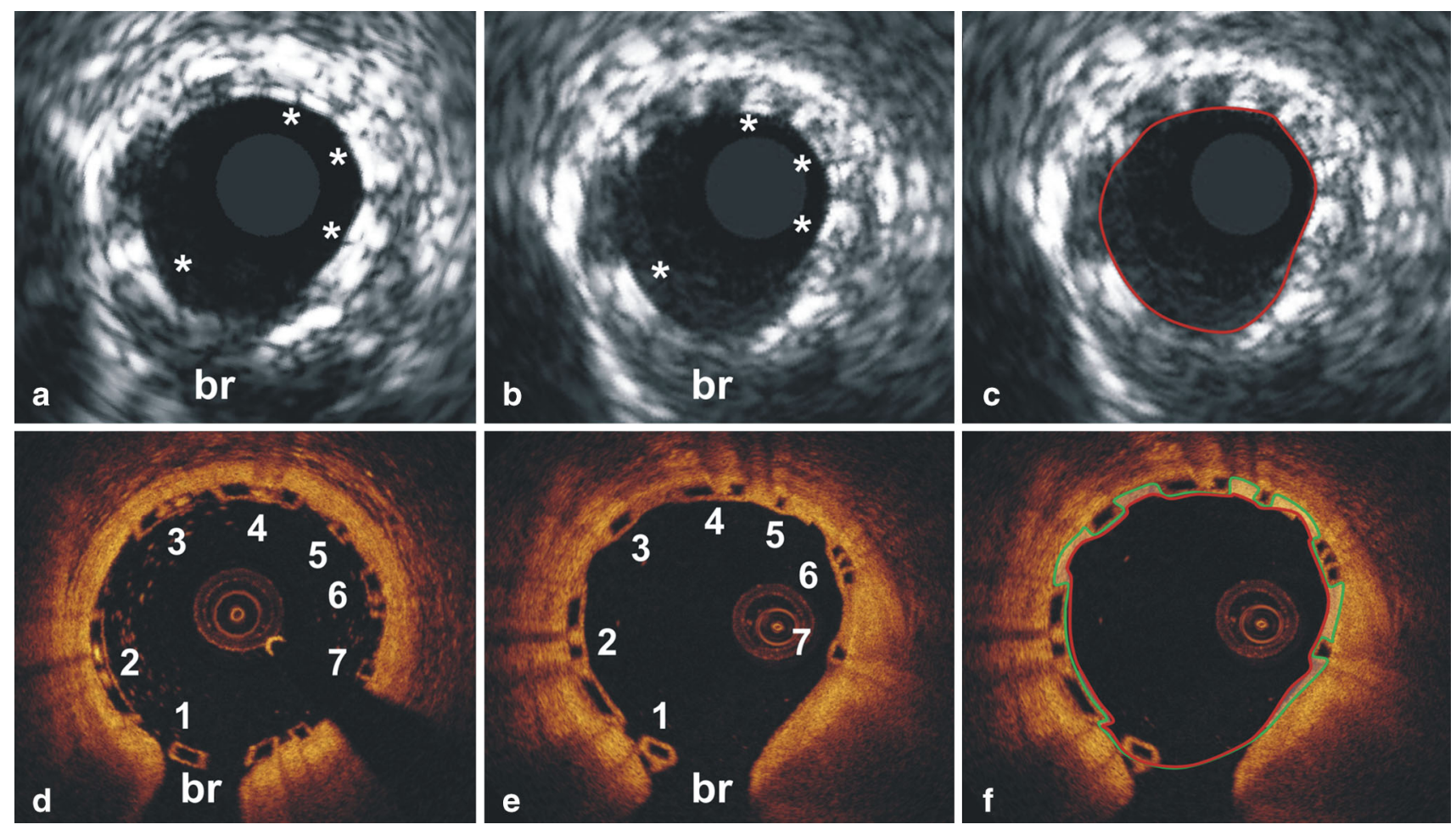

Fig. 1 Intravascular ultrasound (IVUS) and optical coherence tomographic (OCT) images acquired at baseline and the corresponding frames obtained at 6 months follow-up. The correspondence can be confirmed by the presence of a side branch $(b r)$ in all images. In IVUS the scaffold struts (indicated with an asterisk) appear embedded into the vessel wall at baseline (a) while at 6 months follow-up there was no neointimal tissue (b). Thus, in this frame the scaffold and the lumen border is the same and is delineated by a curve connecting the adluminal side of the scaffold struts (c). On the other hand OCT allows more detailed visualization of the scaffold struts enabling identification of 7 corresponding struts between baseline (d) and follow-up (e) examinations. Two struts at baseline did not have correspondence at follow-up; in addition the circumferential location of the corresponding struts is slightly different in the follow-up frame comparing to baseline. All the struts protruded into the lumen at baseline and thus the scaffold border was defined by the adluminal sides of the struts and between struts by the segment connecting the abluminal side of adjacent struts (e). The area between the luminal border at baseline (green line) and the luminal border at follow-up (red line) corresponds to the neointima tissue that is portrayed in panel (f) in a semi-transparent fashion

defined by the adluminal sides of the struts and between struts by the segment connecting the abluminal side of adjacent struts (Fig. 1). For $68.3 \%$ of the struts analyzed at follow-up the operators were able to identify corresponding struts at the baseline examinations. For the remaining we assumed the struts protruded at baseline since the majority of the struts $(\mathrm{n}=5,700,91.1 \%$ of the detected struts) protruded at baseline. Malapposed struts at follow-up were not taken into consideration and in these segments the baseline luminal border was approximated by the endoluminal border of the neointima.

\section{Reconstruction of coronary anatomy}

The processed IVUS and OCT images were fused separately with the angiographic data using a previously described methodology [6, 10]. In brief this approach includes the extraction of the luminal centerline of the segment of interest from the selected end-diastolic 
angiographic projections, the placement of the detected IVUS/OCT borders onto the centerline, and the estimation of their absolute orientation using efficient algorithms and anatomical landmarks seen in both the angiographic and intravascular imaging data [6, 10].

The outcome of this process was four point clouds (two derived from the IVUS and two from the FD-OCT data) for each studied segment, which were used to construct four non-uniform rational B-spline surfaces. Two surfaces corresponded to the luminal surface at baseline (one is the IVUS-based and the other the OCT-based luminal surface) and were defined by the luminal borders in the non-scaffolded segment and by the scaffold borders in the scaffolded segment whereas the other two surfaces corresponded to the luminal surfaces at follow-up (one is the IVUS-based and the other the OCT-based luminal surface at follow-up) and were defined by the luminal borders in the scaffolded segment.

\section{Blood flow simulation}

The two baseline luminal surfaces derived by the fusion of IVUS and FD-OCT with the angiographic data were further processed with computational fluid dynamics techniques which allowed generation of a finite volume mesh and blood flow simulation by solving the 3D Navier-Stokes equations (ICEM CFD and CFX 11, Ansys, Canonsburg, PA) [11]. To evaluate the detailed characteristics of the hemodynamic micro-environment, the mesh density was increased around the stent struts and within the boundary layer of the flow field, and had a maximum element edge equal to approximately $1 / 4$ of the BVS strut thickness (i.e., $\sim 40 \mu \mathrm{m})$. Blood was regarded as a homogeneous, Newtonian fluid with a dynamic viscosity of $0.0035 \mathrm{~Pa} \mathrm{~s}$ and a density of $1,050 \mathrm{~kg} / \mathrm{m}^{3}$. Blood flow was considered to be steady, laminar and incompressible, and a flat velocity profile was imposed at the inlet of the reconstructed segment. Blood flow for each reconstruction was estimated by measuring in the two angiographic projections, the number of frames required for the contrast agent to pass from the inlet to the outlet of the reconstructed segment, the volume of the reconstructed segment and the cine frame rate [2, 12]. The arterial wall was considered to be rigid and no-slip conditions were applied at the scaffold surface, while zero pressure conditions were imposed at the outlet. ESS at the baseline luminal surface was calculated as the product of blood viscosity and the gradient of blood velocity at the wall.

Data analysis and statistics

The lumen volume of the scaffolded segment at baseline and follow-up were calculated in the IVUS-based and FD-
OCT-based reconstructions and the neointimal volume was defined as: lumen volume at baseline minus lumen volume at follow-up. The percentage scaffold volume obstruction was given by the equation: $100 \times$ neointima volume/ lumen volume at baseline. The NT was defined as the distance between the luminal surface at baseline and follow-up and was measured around the circumference of the lumen per $5^{\circ}$ interval and along the axial direction per $0.2 \mathrm{~mm}$ interval with the use of an in-house algorithm developed in Visual Fortran (Compaq Computer Corporation, Houston, TX, USA) [11]. The NT was related to the ESS estimated at the baseline luminal surface at the corresponding location. Segments within the scaffold located at the origin of side branches and their adjacent segments with length equal to the diameter of the side branch were excluded from the analysis since the branches were not included in the 3D reconstruction, and thus the computed ESS in these areas was not considered to be reliable. In addition, the $2.5 \mathrm{~mm}$ proximal and distal end of the scaffold was also excluded from the analysis.

Continuous variables are reported as mean and standard deviation, as normality was not rejected by the Kolmogorov-Smirnov test. Categorical variables are presented as counts and percentages. Comparison between continuous variables was performed using the paired or unpaired $t$ test depending on their association. Pearson correlation coefficient and linear regression analysis were implemented to investigate the association between the logarithmic transformed baseline ESS (lnESS) and the estimated NT at follow-up. To control for patient effect, a mixed model with random intercept and slope was used to estimate the overall association between lnESS and NT in the IVUS-based and the FDOCT-based reconstructions. In this mixed model, the autoregressive covariance structure was utilized to take into consideration the nested structure of cross-sections within subjects.

A $P$ value $<0.05$ was considered statistical significant. Statistical analysis was performed with the SPSS statistical software package (version 18.0 for Windows, SPSS, Inc, Chicago, IL, USA) and the SAS software (version 9.2, SAS Institute Inc, Cary, NC, USA).

\section{Results}

Patient characteristics

Five patients (5 scaffolded segments) from Cohort B1 had biplane coronary angiography, IVUS, and FD-OCT at 6 months follow-up and 1 patient (1 scaffolded segment) from Cohort B2 at 12 months follow-up and were included in the current analysis. The baseline characteristics of the 
Table 1 Baseline characteristics of the studied population

\begin{tabular}{ll}
\hline & $\begin{array}{l}\text { Studied population } \\
(\mathrm{n}=6)\end{array}$ \\
\hline Baseline characteristics & \\
Age (years) & $57 \pm 6$ \\
Male & $5(83 \%)$ \\
Hypertension & $3(50 \%)$ \\
Hypercholesterolemia & $6(100 \%)$ \\
Diabetes mellitus & $0(0 \%)$ \\
Current smoking & $0(0 \%)$ \\
Prior percutaneous coronary intervention & $1(17 \%)$ \\
Prior myocardial infarction & $1(17 \%)$ \\
Stable angina & $6(100 \%)$ \\
Left anterior descending artery & $3(50 \%)$ \\
Left circumflex artery & $1(17 \%)$ \\
Right coronary artery & $2(33 \%)$ \\
Ramus intermedius & $0(0 \%)$ \\
Medications & \\
$\beta$-blockers & $3(50 \%)$ \\
Renin-angiotensin aldosterone & $3(50 \%)$ \\
inhibitors & \\
Statins & $6(100 \%)$ \\
\hline
\end{tabular}

studied patients and the studied segments are shown in Table 1.

\section{Coronary reconstruction analysis}

The length of the reconstructed segments was $35.7 \pm 4.8 \mathrm{~mm}$ whereas the length of the scaffolded segments was $19.7 \pm 3.0 \mathrm{~mm}$ in the IVUS-based and $19.4 \pm 0.6 \mathrm{~mm}$ in the FD-OCT-based reconstructions $(P=0.815)$. The baseline lumen volume of the scaffolded segment was increased in the FD-OCT-based models comparing to the IVUS-based models but there was no difference at follow-up. IVUS imaging identified neointimal proliferation in 5 of the 6 scaffolded segments (in only $8.6 \%$ of the analyzed IVUS frames) while FD-OCT detected neointimal tissue in all the treated segments. IVUS was unable to accurately estimate neointimal formation as the percentage scaffold volume obstruction and the mean NT was considerably lower in the IVUSbased comparing to the FD-OCT-based reconstructions (Table 2).

\section{Shear stress analysis}

The mean ESS (scaffold level analysis) was considerably higher in the scaffolded segments in the IVUS-based reconstructions compared to the FD-OCT-based reconstructions $(1.87 \pm 0.66$ vs. $1.29 \pm 0.66 \mathrm{~Pa}, P=0.030)$.
Table 2 Differences between the IVUS-based and FD-OCT-based reconstructed models with regards the scaffolded segments

\begin{tabular}{lccc}
\hline & $\begin{array}{l}\text { IVUS-based } \\
\text { models }(\mathrm{n}=6)\end{array}$ & $\begin{array}{l}\text { FD-OCT } \\
\text { models }(\mathrm{n}=6)\end{array}$ & $P$ \\
\hline $\begin{array}{c}\text { Baseline mean lumen } \\
\text { area }\left(\mathrm{mm}^{2}\right)\end{array}$ & $6.88 \pm 1.81$ & $7.75 \pm 1.24$ & 0.561 \\
$\begin{array}{c}\text { Follow-up mean } \\
\text { lumen area }\left(\mathrm{mm}^{2}\right)\end{array}$ & $6.85 \pm 1.81$ & $6.97 \pm 0.99$ & 0.764 \\
$\begin{array}{c}\text { Mean neointima } \\
\text { tissue area }\left(\mathrm{mm}^{2}\right)\end{array}$ & $0.03 \pm 0.03$ & $0.78 \pm 0.29$ & 0.001 \\
$\begin{array}{c}\text { Mean neointima } \\
\text { thickness }(\mu \mathrm{m})\end{array}$ & $14 \pm 13$ & $102 \pm 29$ & $<0.0001$ \\
$\begin{array}{c}\text { Lumen volume at } \\
\left.\text { baseline (mm }{ }^{3}\right)\end{array}$ & $132.83 \pm 35.85$ & $150.43 \pm 24.96$ & 0.078 \\
$\begin{array}{c}\text { Lumen volume at } \\
\left.\text { follow-up (mm }{ }^{3}\right)\end{array}$ & $132.16 \pm 25.47$ & $135.29 \pm 20.44$ & 0.656 \\
$\begin{array}{c}\text { Neointima tissue } \\
\text { volume }\left(\mathrm{mm}^{3}\right)\end{array}$ & $0.67 \pm 0.59$ & $15.14 \pm 5.63$ & 0.001 \\
$\begin{array}{c}\text { Percentage volume } \\
\text { obstruction }(\%)\end{array}$ & $0.47 \pm 0.39$ & $9.86 \pm 2.39$ & $<0.0001$ \\
\hline
\end{tabular}

Twenty six percent of the ESS estimated in the IVUSbased models and $43 \%$ of the ESS estimated in the FDOCT models was $<1 \mathrm{~Pa}$ (i.e., low ESS with a pro-restenotic effect) [13]. This difference should be attributed not only to the increased baseline luminal dimensions reported in the FD-OCT-derived reconstructions but also to the fact that FD-OCT imaging allowed detailed assessment of the lumen and struts and evaluation of the irregular luminal morphology created from the protruding struts that affected the local hemodynamic micro-environment. As it is shown in Fig. 2, ESS was increased on the top of the struts whereas in the between the struts areas there were flow recirculation zones (especially distally to the struts) and low ESS values.

The differences in the computed ESS at baseline and NT at follow-up between the IVUS-based and FD-OCT-based reconstructions had a considerable effect on the association between these two variables. A negative correlation between baseline lnESS and NT at follow-up was reported in both IVUS- and FD-OCT-based models (mean correlation coefficient: $-0.10 \pm 0.04$ vs. $-0.52 \pm 0.19$, $P=0.028$ respectively); however the magnitude of the estimates of the intercepts and slopes was quite different. Table 3 provides the correlation coefficients, as well as the estimated slopes and y-intercepts after applying linear mixed regression analysis for each subject (Fig. 3). The overall linear mixed-effect model with follow-up time included as a fixed effect yielded in the IVUS-based reconstructions an overall slope of $-1 \mu \mathrm{m} / \mathrm{ln}(\mathrm{Pa})$ and $y$-intercept of $2 \mu \mathrm{m}$ while in the OCT-based reconstructions the overall slope was of $-32 \mu \mathrm{m} / \ln (\mathrm{Pa})$ and y-intercept of $76 \mu \mathrm{m}$ [slope difference: $31 \mu \mathrm{m} / \ln (\mathrm{Pa})$; 


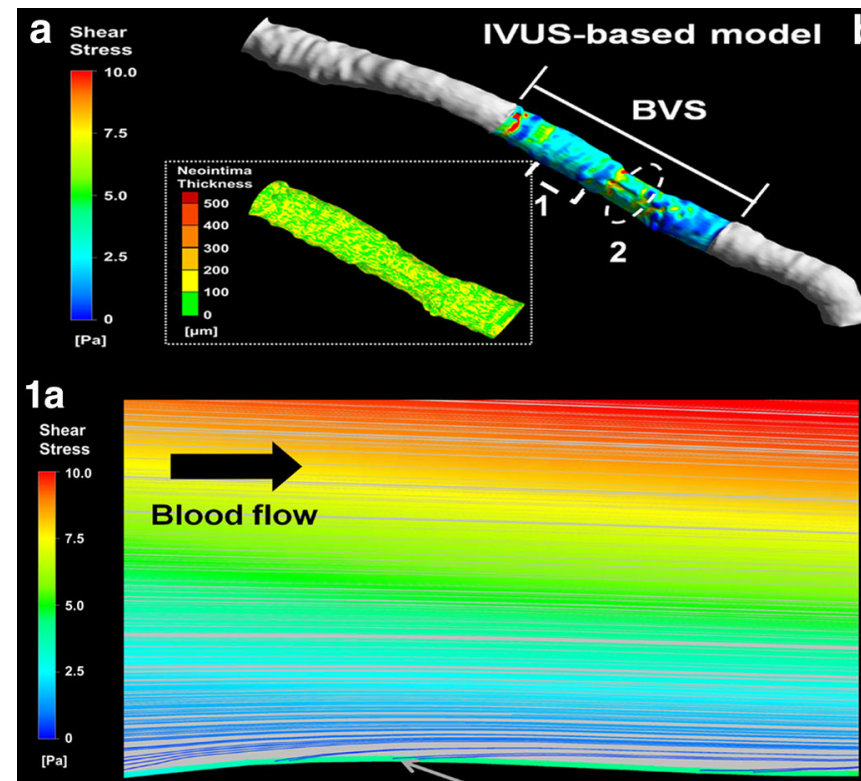

Baseline lumen surface
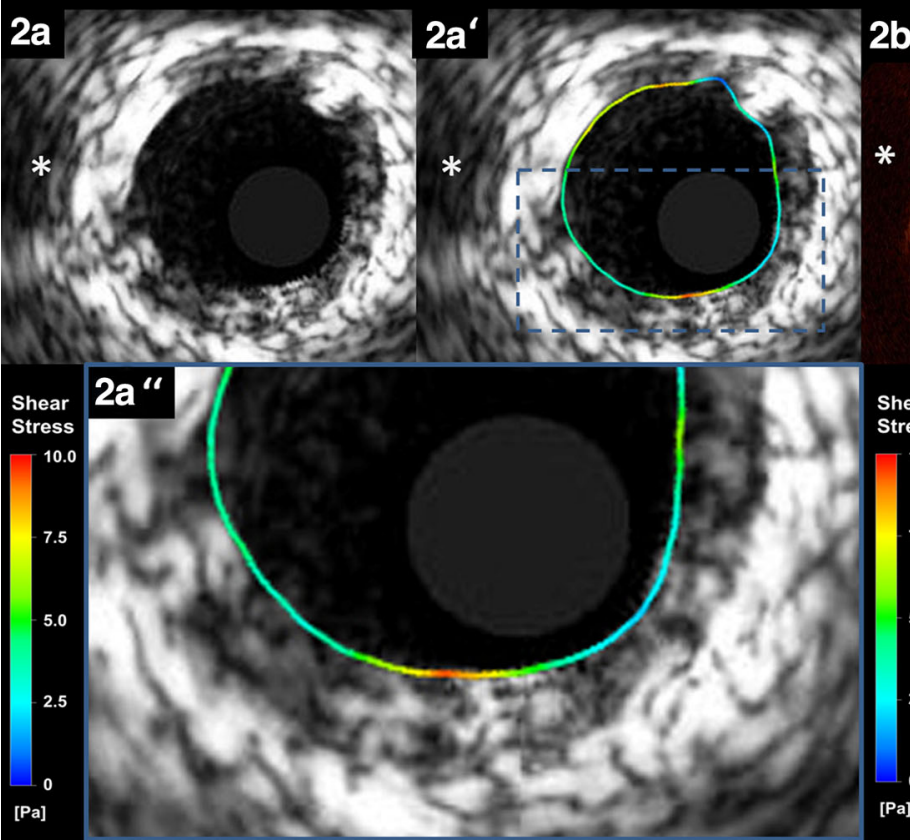

Fig. 2 Three dimensional reconstruction of the baseline luminal surface by fusing intravascular ultrasound (IVUS) with angiographic data (a) and optical coherence tomographic (OCT) and angiographic data (b). The endothelial shear stress (ESS) in the scaffolded segment is portrayed with the use of a color coded map with the blue color corresponding to low and the red to high ESS. The insets demonstrate the distribution of the neointima tissue developed at follow-up (green color indicates no neointima and red increased neointima thickness). Panels 1a and $1 \mathrm{~b}$ show longitudinal cross sections of the reconstructed baseline surfaces with the blood flow streamlines portrayed with velocity color coding. In the IVUS-based model there is no neointima tissue, no flow disruption, and the ESS has a homogeneous distribution. On the other hand in the OCT-based model the protruding struts create flow disturbances with low velocities and recirculation zones in the between the struts areas. ESS is low in these regions and increased

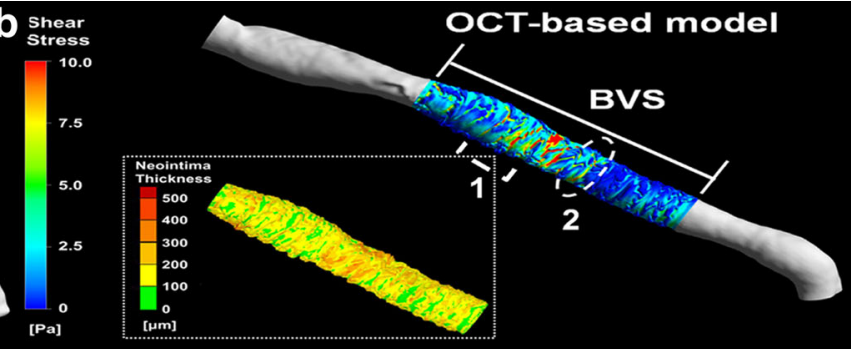

16

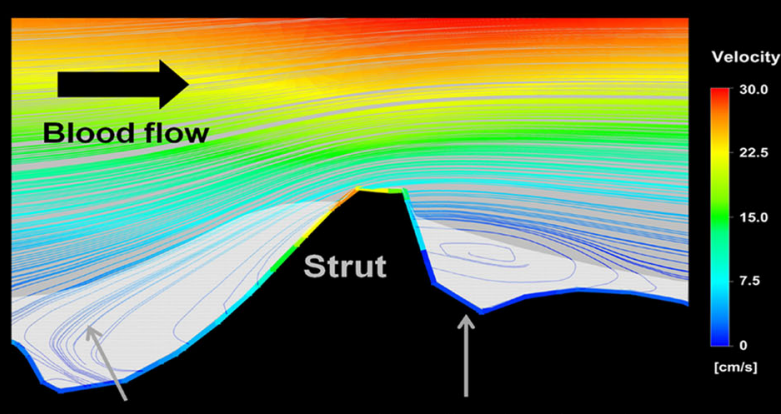

Neointima tissue Baseline luminal surface

2b
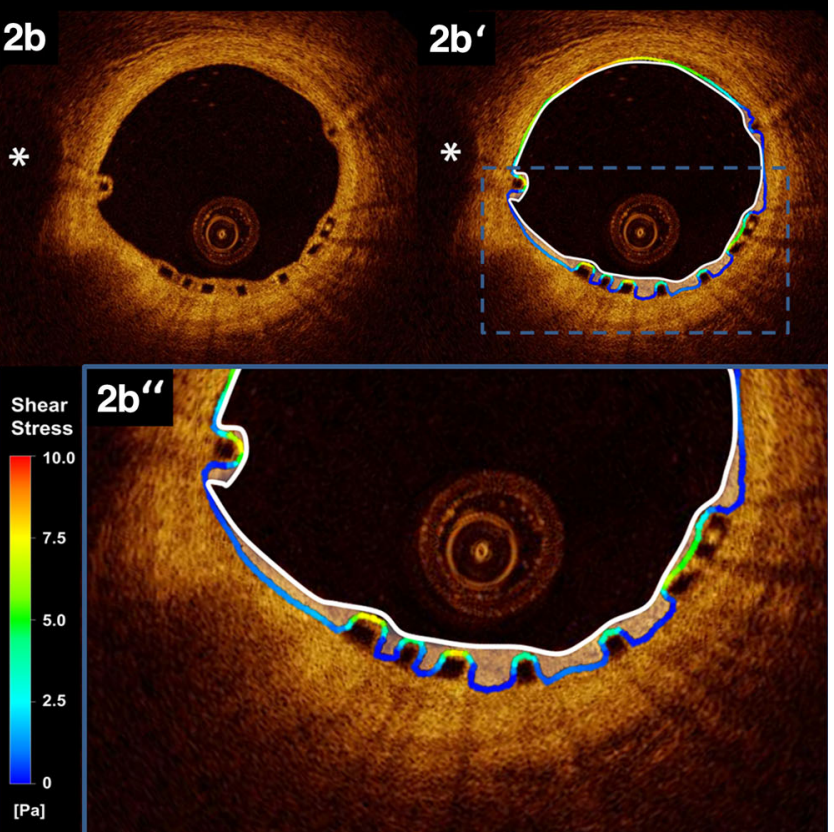

on the top of the struts. There appears to be an inverse association between the neointimal tissue, portrayed in transparent fashion, and the baseline ESS as there is increased neointima tissue in the segments between the struts and no neointimal on the top of the struts. Similar findings are shown in the IVUS and OCT cross sections acquired at the middle of the scaffolded segment just before the origin of a side branch (indicated with an asterisk). The luminal surface in the IVUS frame appears smooth, there is no neointima tissue at follow-up, while ESS has a homogeneous distribution (2a, 2a', 2a' ${ }^{\prime \prime}$. On the other hand, OCT allows more detailed assessment of the rugged luminal morphology created post scaffold implantation and permits detection of the neointimal tissue developed at follow-up ( $2 b$, $\left.2 b^{\prime}, 2 b^{\prime \prime}\right)$. The ESS at the baseline luminal surfaces has a heterogeneous distribution with increased ESS on the top of the struts and low ESS values in the between the struts areas 
Table 3 Correlation coefficient, slope and y-intercept obtained after applying linear mixed regression analysis between logarithmic transformed baseline ESS (lnESS) and neointima thickness in the IVUS-based and FD-OCT-based reconstructions

\begin{tabular}{|c|c|c|c|c|c|c|c|}
\hline \multirow{2}{*}{$\begin{array}{l}\text { Patient } \\
\text { number }\end{array}$} & \multirow{2}{*}{$\begin{array}{l}\text { Follow-up period } \\
\text { (months) }\end{array}$} & \multicolumn{3}{|c|}{ IVUS-based-reconstructions } & \multicolumn{3}{|c|}{ FD-OCT-based-reconstructions } \\
\hline & & $\begin{array}{l}\text { Correlation } \\
\text { coefficient }\end{array}$ & $\begin{array}{l}\text { Slope } \\
(\mu \mathrm{m} /(\ln ) \mathrm{Pa})\end{array}$ & $\begin{array}{l}y \text {-intercept } \\
(\mu \mathrm{m})\end{array}$ & $\begin{array}{l}\text { Correlation } \\
\text { coefficient }\end{array}$ & $\begin{array}{l}\text { Slope } \\
(\mu \mathrm{m} /(\ln ) \mathrm{Pa})\end{array}$ & $\begin{array}{l}\text { y-intercept } \\
(\mu \mathrm{m})\end{array}$ \\
\hline 1 & 6 & -0.043 & -14 & 36 & -0.800 & -60 & 106 \\
\hline 2 & 6 & -0.140 & -8 & 14 & -0.571 & -30 & 71 \\
\hline 3 & 6 & -0.086 & 0 & 0 & -0.451 & -28 & 76 \\
\hline 4 & 6 & -0.124 & -4 & 17 & -0.537 & -35 & 124 \\
\hline 5 & 6 & - & - & - & -0.219 & -12 & 59 \\
\hline 6 & 12 & -0.127 & -11 & 30 & -0.537 & -38 & 99 \\
\hline
\end{tabular}

$P<0.0001]$. In the overall mixed effect model, with fixed effects of modality and follow-up time added, the $P$ values associated with modality, follow-up time, and lnESS were $<0.0001,0.0006$, and $<0.0001$ respectively, indicating statistically significant influence of modality, follow-up time, and $\operatorname{lnESS}$ on NT.

\section{Discussion}

The present analysis compared for the first time the association between ESS and neointimal proliferation following Absorb BVS implantation, in coronary reconstructions obtained either by fusing FD-OCT or IVUS with angiographic data. We found: (1) increased NT in the FD-OCTbased models compared to the IVUS-based models, (2) lower ESS values and flow disturbances in the FD-OCTbased models that were not detected in IVUS-based reconstructions, (3) an inverse and statistical significant correlation between $\operatorname{lnESS}$ and NT in both FD-OCT-based and IVUS-based models, and (4) an effect of the modality used to reconstruct coronary anatomy and simulate blood flow on this association with a higher correlation in the FD-OCTbased reconstructions.

Previous studies investigating the effect of ESS on neointimal proliferation relied on IVUS imaging, a modality with limited resolution which did not allow detailed assessment of lumen morphology and quantification of neointimal tissue especially in segments implanted with drug eluting stents where neointimal proliferation is minimal [1-4]. Thus, the measured ESS in these models, were determined by the macroscopic geometry of the reconstructed artery (i.e., curvature and luminal dimensions) as it was not possible to evaluate the impact of the protruded struts on the local hemodynamic micro-environment [14-16]. Numerous in silico studies have shown that stent design critically determines ESS patterns with the stents having thick rectangularshaped struts connected with perpendicular to the flow direction connectors to modulate a low ESS environment, whereas the devices with thin circular-shaped struts that are connected with parallel to the flow direction connectors to have a minimal effect on the local flow [16-20]. The poor resolution of IVUS imaging and the blooming effect of the metallic struts did not permit meticulous evaluation of the effect of stent implantation on luminal morphology and assessment of the effect of the hemodynamic micro-milieu on neointimal proliferation.

Frequency domain optical coherence tomography overcomes these limitations as it provides realistic 3D models which allow detailed evaluation of the luminal surface, post scaffold implantation, and quantification of NT. We demonstrated that the high resolution FD-OCT-based reconstructions permitted reliable and meticulous 3D representation of the rugged luminal surface, created post Absorb BVS implantation, which appeared to affect the local blood flow patterns. The protruding struts disturbed the blood flow resulting in flow recirculation zones and low ESS between the struts and higher ESS on the top of the struts. The hemodynamic micro-environment had a considerable effect on neointimal formation as we noticed a heterogeneous neointimal distribution with an increased NT in the between struts areas (low ESS) and minimal neointimal tissue over the struts (high ESS) (Fig. 4). Therefore, the correlation between ESS and NT was higher in the FD-OCT-based reconstructions compared to the IVUS-based models, while the mixed linear regression model confirmed the effect of the imaging modality used to examine the association between ESS and NT.

This analysis focused on the association between ESS and NT in segments implanted with an Absorb BVS for the following reasons: (1) in contrast to the metallic stents where there is a blooming effect in OCT and shadowing behind the struts, the struts of the Absorb BVS appear as echolucent boxes allowing accurate delineation of luminal borders, (2) the struts of the Absorb BVS have a heterogenic appearance in 

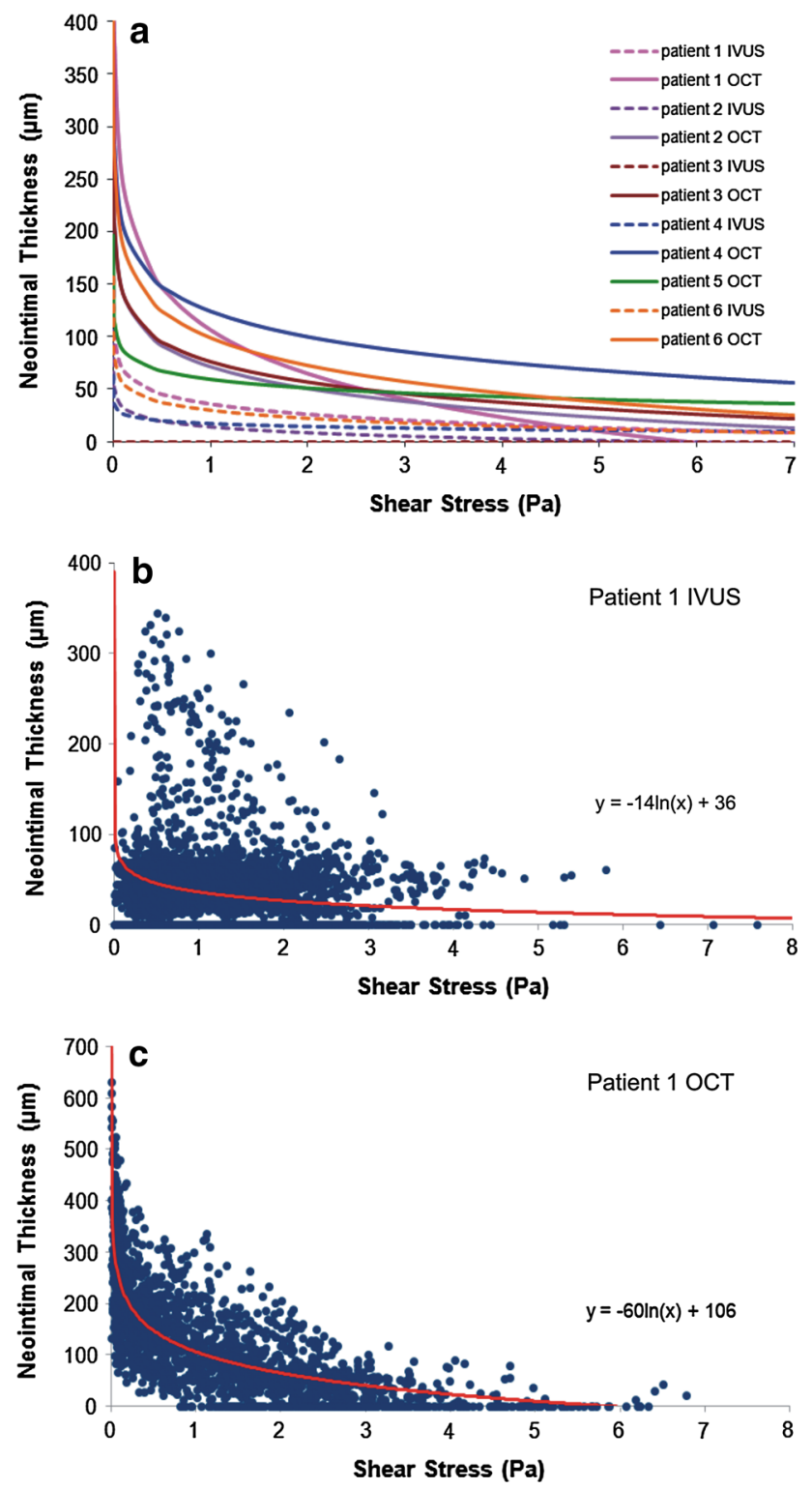

Fig. 3 Regression curves showing the association between ESS (lnESS) at baseline and neointima thickness at follow-up in the 6 intravascular ultrasound (IVUS)-based and optical coherence tomographic (OCT)-based reconstructions (a). Regression analysis between lnESS and neointima thickness for an IVUS-based (b) and OCT-based reconstruction (c)

FD-OCT which permits us to find corresponding struts at baseline and follow-up examinations and use this information to reconstruct in a more accurate fashion the baseline luminal surface, (3) the rectangular shape and increased thickness $(156 \mu \mathrm{m})$ of the scaffold struts as well as the in-phase zig-zag hoops linked with bridges design of the scaffold is anticipated to influence considerably the local flow and ESS patterns, and (4) bioresorbable devices may gradually lose their geometrical integrity and tend to expand; however the changes in scaffold geometry and dimensions appear to occur after the first year post device implantation and thus we can assume with some certainty that the scaffold geometry at 6 months and 1 year follow-up is not different from its geometry at baseline post device implantation [8, 21, 22]. The fact that Absorb BVS allows accurate FD-OCT-based luminal reconstruction and evaluation of the effect of the hemodynamic micro-milieu on neointimal formation enables us to reveal the limitations of IVUS-derived reconstructions in assessing the effect of ESS on NT.

To our knowledge this is the first study that provides evidence about the superiority of FD-OCT-based over the IVUS-based models in assessing the implications of the local hemodynamic environment on neointimal proliferation. Numerous in silico blood flow studies have demonstrated the implications of different stent designs on the local ESS patterns, but no study up to date has evaluated these effects in vivo [16-20]. The FD-OCT-based reconstruction appears to enable assessment of the hemodynamic micro-environment and thus it is anticipated to be proven useful in the future to investigate in vivo the athero-promoting and prothrombotic implications of different stent designs.

\section{Limitations}

A considerable limitation of the current analysis is the small number of patients included. However, the increased number of measurements (approximately 6,480 estimations per scaffold in each reconstruction) provides us enough power to compare the estimations of the two approaches and draw solid conclusions. Another limitation of the analysis was the fact that we used the follow-up data to reconstruct both the luminal surface at baseline and followup. This was done because there were considerable differences in the corresponding FD-OCT frames with regards the number of the portrayed struts and the circumferential location of corresponding struts (Fig. 1), that did not allow us to accurately associate the luminal surface reconstructed from the baseline FD-OCT data with the luminal surface reconstructed from the follow-up data and estimate the NT. Malapposed struts at follow-up were not taken into account in this analysis. However, considering the low malapposition rate reported at follow-up in Absorb Cohort B study at 6 months follow-up $(0.7 \%)$ it is unlikely that this approximation affected our results [9]. Finally, it should be mentioned that the abovementioned analysis cannot be performed in metallic stents because: (1) the metallic struts have a homogenous appearance and thus it is almost impossible to identify corresponding struts between baseline and follow-up and (2) the blooming artefacts makes often difficult the discrimination between protruded and embedded struts. 

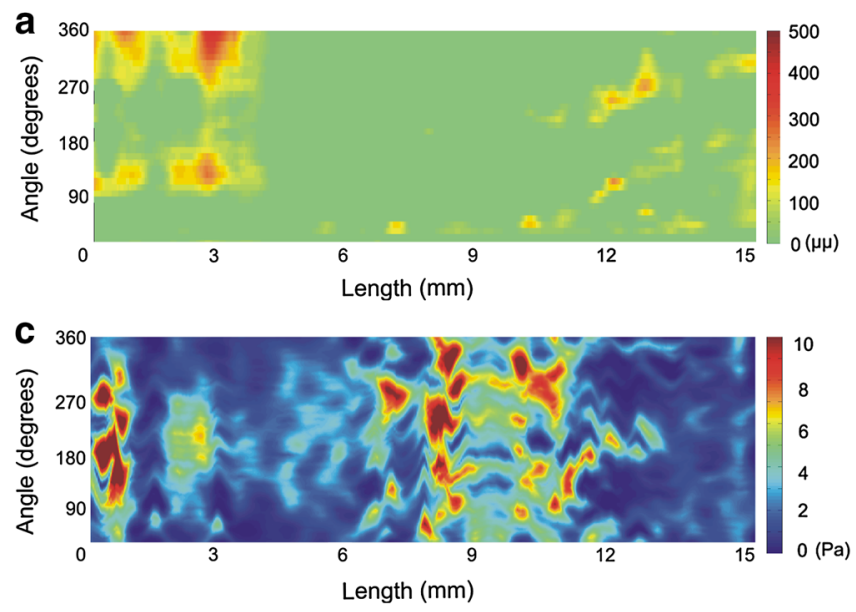

Fig. 4 Spread-out plots showing the neointima and the endothelial shear stress (ESS) distribution in the intravascular ultrasound (IVUS) - (a, c) and the optical coherence tomography (OCT)-based

\section{Conclusions}

This study demonstrated for the first time that the fusion of FD-OCT and coronary angiographic data provides more detailed reconstruction of luminal morphology in segments implanted with an Absorb BVS and accurate evaluation of the effect of ESS on vessel wall healing than the IVUSderived models. The ability of FD-OCT to assess the hemodynamic micro-environment in treated segments is anticipated to allow us to investigate in vivo the prorestenotic and pro-thrombotic implications of different stent designs.

Acknowledgments The first author is funded by the Hellenic Cardiological Society, Athens, Greece.

Conflict of interest Jin Wang is employee of Abbott Vascular. None of the other authors have any conflict of interest to declare.

\section{References}

1. Gijsen FJ, Oortman RM, Wentzel JJ, Schuurbiers JC, Tanabe K, Degertekin M, Ligthart JM, Thury A, de Feyter PJ, Serruys PW, Slager CJ (2003) Usefulness of shear stress pattern in predicting neointima distribution in sirolimus-eluting stents in coronary arteries. Am J Cardiol 92(11):1325-1328

2. Papafaklis MI, Bourantas CV, Theodorakis PE, Katsouras CS, Naka KK, Fotiadis DI, Michalis LK (2010) The effect of shear stress on neointimal response following sirolimus- and paclitaxeleluting stent implantation compared with bare-metal stents in humans. JACC Cardiovasc Interv 3(11):1181-1189. doi:10.1016/ j.jcin.2010.08.018

3. Suzuki N, Nanda H, Angiolillo DJ, Bezerra H, Sabate M, Jimenez-Quevedo P, Alfonso F, Macaya C, Bass TA, Ilegbusi OJ, Costa MA (2008) Assessment of potential relationship between wall shear stress and arterial wall response after bare metal stent and sirolimus-eluting stent implantation in patients with diabetes
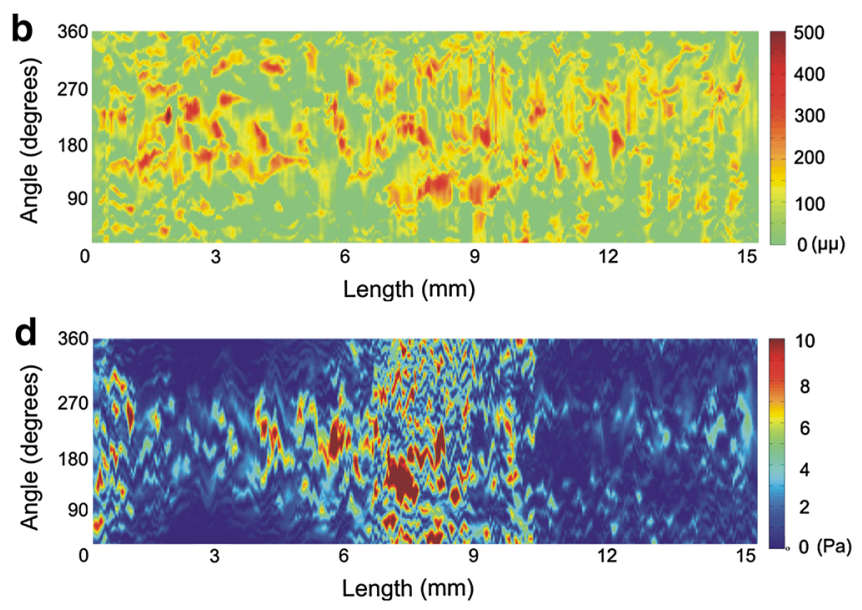

models (b, d). The ESS and the neointima has a homogeneous distribution in the IVUS based models while in the OCT based reconstructions the ESS and the neointima appears heterogeneous

mellitus. Int J Cardiovasc Imaging 24(4):357-364. doi:10.1007/ s10554-007-9274-0

4. Wentzel JJ, Krams R, Schuurbiers JC, Oomen JA, Kloet J, van Der Giessen WJ, Serruys PW, Slager CJ (2001) Relationship between neointimal thickness and shear stress after Wallstent implantation in human coronary arteries. Circulation 103(13):1740-1745

5. Guagliumi G, Sirbu V (2008) Optical coherence tomography: high resolution intravascular imaging to evaluate vascular healing after coronary stenting. Catheter Cardiovasc Interv 72(2):237-247. doi:10.1002/ccd.21606

6. Athanasiou LS, Bourantas CV, Siogkas PK, Sakellarios AI, Exarchos TP, Naka KK, Papafaklis MI, Michalis LK, Prati F, Fotiadis DI (2012) 3D reconstruction of coronary arteries using frequency domain optical coherence tomography images and biplane angiography. Conf Proc IEEE Eng Med Biol Soc 2012:2647-2650. doi:10.1109/EMBC.2012.6346508

7. Papafaklis MI, Bourantas CV, Yonetsu T, Kato K, Kotsia A, Coskun AU, Jia H, Antoniadis AP, Vergallo R, Tsuda M, Fotiadis DI, Feldman CL, Stone PH, Jang IK, Michalis LK (2012) Geometrically accurate three-dimensional coronary artery reconstruction using frequency-domain optical coherence tomography and angiographic data: new opportunities for in vivo endothelial shear stress assessment. JACC Cardiovasc Interv 6:S36

8. Serruys PW, Onuma Y, Ormiston JA, de Bruyne B, Regar E, Dudek D, Thuesen L, Smits PC, Chevalier B, McClean D, Koolen J, Windecker S, Whitbourn R, Meredith I, Dorange C, Veldhof S, Miquel-Hebert K, Rapoza R, Garcia-Garcia HM (2010) Evaluation of the second generation of a bioresorbable everolimus drug-eluting vascular scaffold for treatment of de novo coronary artery stenosis: six-month clinical and imaging outcomes. Circulation 122(22):2301-2312. doi:10.1161/CIRCU LATIONAHA.110.970772

9. Gomez-Lara J, Radu M, Brugaletta S, Farooq V, Diletti R, Onuma Y, Windecker S, Thuesen L, McClean D, Koolen J, Whitbourn R, Dudek D, Smits PC, Regar E, Veldhof S, Rapoza R, Ormiston JA, Garcia-Garcia HM, Serruys PW (2011) Serial analysis of the malapposed and uncovered struts of the new generation of everolimus-eluting bioresorbable scaffold with optical coherence tomography. JACC Cardiovasc Interv 4(9):992-1001. doi:10.1016/j.jcin.2011.03.020

10. Bourantas CV, Papafaklis MI, Athanasiou L, Kalatzis FG, Naka KK, Siogkas PK, Takahashi S, Saito S, Fotiadis DI, Feldman CL, 
Stone PH, Michalis LK (2013) A new methodology for accurate 3-dimensional coronary artery reconstruction using routine intravascular ultrasound and angiographic data: implications for widespread assessment of endothelial shear stress in humans. EuroIntervention 9(5):582-93. doi:10.4244/EIJV9I5A94

11. Papafaklis MI, Bourantas CV, Theodorakis PE, Katsouras CS, Fotiadis DI, Michalis LK (2009) Relationship of shear stress with instent restenosis: bare metal stenting and the effect of brachytherapy. Int J Cardiol 134(1):25-32. doi:10.1016/j.ijcard.2008.02.006

12. Sakamoto S, Takahashi S, Coskun AU, Papafaklis MI, Takahashi A, Saito S, Stone PH, Feldman CL (2013) Relation of distribution of coronary blood flow volume to coronary artery dominance. Am J Cardiol. doi:10.1016/j.amjcard.2013.01.290

13. Stone PH, Saito S, Takahashi S, Makita Y, Nakamura S, Kawasaki T, Takahashi A, Katsuki T, Namiki A, Hirohata A, Matsumura T, Yamazaki S, Yokoi H, Tanaka S, Otsuji S, Yoshimachi F, Honye J, Harwood D, Reitman M, Coskun AU, Papafaklis MI, Feldman CL (2012) Prediction of progression of coronary artery disease and clinical outcomes using vascular profiling of endothelial shear stress and arterial plaque characteristics: the PREDICTION Study. Circulation 126(2):172-181. doi:10.1161/ CIRCULATIONAHA.112.096438

14. He Y, Duraiswamy N, Frank AO, Moore JE Jr (2005) Blood flow in stented arteries: a parametric comparison of strut design patterns in three dimensions. J Biomech Eng 127(4):637-647

15. Balossino R, Gervaso F, Migliavacca F, Dubini G (2008) Effects of different stent designs on local hemodynamics in stented arteries. J Biomech 41(5):1053-1061. doi:10.1016/j.jbiomech. 2007.12.005

16. Duraiswamy N, Schoephoerster RT, Moore JE Jr (2009) Comparison of near-wall hemodynamic parameters in stented artery models. J Biomech Eng 131(6):061006. doi:10.1115/1.3118764
17. Jimenez JM, Davies PF (2009) Hemodynamically driven stent strut design. Ann Biomed Eng 37(8):1483-1494. doi:10.1007/ s10439-009-9719-9

18. Mejia J, Ruzzeh B, Mongrain R, Leask R, Bertrand OF (2009) Evaluation of the effect of stent strut profile on shear stress distribution using statistical moments. Biomed Eng Online 8:8. doi:10.1186/1475-925X-8-8

19. LaDisa JF Jr, Olson LE, Guler I, Hettrick DA, Kersten JR, Warltier DC, Pagel PS (2005) Circumferential vascular deformation after stent implantation alters wall shear stress evaluated with timedependent 3D computational fluid dynamics models. J Appl Physiol 98(3):947-957. doi:10.1152/japplphysiol.0 0872.2004

20. Pant S, Bressloff NW, Forrester AI, Curzen N (2010) The influence of strut-connectors in stented vessels: a comparison of pulsatile flow through five coronary stents. Ann Biomed Eng 38(5):1893-1907. doi:10.1007/s10439-010-9962-0

21. Serruys PW, Onuma Y, Dudek D, Smits PC, Koolen J, Chevalier B, de Bruyne B, Thuesen L, McClean D, van Geuns RJ, Windecker S, Whitbourn R, Meredith I, Dorange C, Veldhof S, Hebert KM, Sudhir K, Garcia-Garcia HM, Ormiston JA (2011) Evaluation of the second generation of a bioresorbable everolimus-eluting vascular scaffold for the treatment of de novo coronary artery stenosis: 12-month clinical and imaging outcomes. J Am Coll Cardiol 58(15):1578-1588. doi:10.1016/j.jacc.2011.05.050

22. Ormiston JA, Serruys PW, Onuma Y, van Geuns RJ, de Bruyne B, Dudek D, Thuesen L, Smits PC, Chevalier B, McClean D, Koolen J, Windecker S, Whitbourn R, Meredith I, Dorange C, Veldhof S, Hebert KM, Rapoza R, Garcia-Garcia HM (2012) First serial assessment at 6 months and 2 years of the second generation of absorb everolimus-eluting bioresorbable vascular scaffold: a multi-imaging modality study. Circ Cardiovasc Interv 5(5):620-632. doi:10.1161/CIRCINTERVENTIONS.112.971549 